\title{
EL MODELO DE DEMANDAS-CONTROL-APOYO Y SU RELACIÓN CON EL RIESGO PERCIBIDO DE ENFERMEDAD-ACCIDENTE Una aplicación a la Comunidad Autónoma andaluza
}

\author{
THE JOB DEMANDS-CONTROL-SUPPORT MODEL AND PERCEIVED \\ RISK OF ILLNESS OR ACCIDENT \\ An application to Andalusia (Spain)
}

MARía JosÉ MonTERo Simó jmontero@etea.com
Universidad Loyola Andalucía. España

PILAR RIvera ToRRes privera@unizar.es

Universidad de Zaragoza. España

Rafael Ángel Araque Padilla raraque@etea.com

Universidad Loyola Andalucía. España

\begin{abstract}
RESUMEN
El presente trabajo analiza de qué modo los componentes del modelo de Demandas-Control-Apoyo-JDC(-S) influyen en el riesgo percibido de enfermedad o accidente en el lugar de trabajo y, en concreto, el papel del Control y del Apoyo como variables moduladoras entre las Demandas y el Riesgo percibido. Para ello se ha analizado la estructura dimensional de las componentes de dicho modelo a partir de la Primera Encuesta Andaluza de Condiciones de Trabajo. La muestra final está formada por 7.512 trabajadores y la aproximación metodológica se concreta en los Modelos de Ecuaciones Estructurales con Variables Latentes. Los resultados indican que cabe diferenciar cuatro dimensiones en dicho modelo, de las cuales únicamente tres se muestran condicionantes del Riesgo percibido (Demanda Cuantitativa, Control y Apoyo). Paralelamente, no se obtiene evidencia que sustente la modulación del Control y del Apoyo entre las Demandas y el Riesgo percibido.
\end{abstract}

\section{Palabras Clave}

Encuesta andaluza de condiciones de trabajo; Modelo de Demandas-Control-Apoyo (Modelo JDC(-S)); Modelos de ecuaciones estructurales con variables latentes; Riesgo percibido.

\section{AbstRact}

This paper analyzes how the Job Demand-Control-Support Model (JDC (-S) Model) components influence the risk perception of illness or accident in the workplace and, in particular, the role of Control and Support as moderator variables between the demands and the risk perception. For this purpose, we have analyzed the dimensional structure of the components of the said model from the First Andalusian Labour Conditions Survey. The final sample consists of 7,512 employees and the methodological approach is based on the Structural Equation Models with Latent Variables. Results show four different dimensions in this model, only three of which are shown to influence risk perception (Quantitative Demand, Control and Support). Additionally, no evidence is found to support the modulation of Control and Support between Demands and risk perception.

\section{KEYWORDS}

Job Demand-Control-Support Model (JDC(-S) Model); Perceived Risk; Andalusian Job Conditions Survey 2008; SEM. 


\section{INTRODUCCIÓN}

El Modelo Demandas-Control (JDC) —Karasek 1979— se distingue de otros modelos de estrés laboral por su simplicidad y por el alcance que ha tenido en investigación y predicciones en relación con dos tipos diferentes de resultados: problemas de salud y comportamientos. El modelo JDC desarrollaba la corriente de estudio sobre el rediseño del puesto de trabajo, añadiendo a las variables habituales de autonomía, satisfacción, motivación y compromiso, los efectos sobre la salud del trabajador, combinando así el enfoque tradicional de la psicología organizacional con el de las Demandas del trabajo o estresores. De manera que el modelo JDC, y su ampliación posterior al Modelo Demandas-Control-Apoyo (JDC(-S)) - Johnson y Hall 1988- se ha utilizado para explicar problemas de salud en el trabajo como: cansancio (Akerboom y Maes 2006; Rafferty, Friend y Landsbergis 2001; Karasek 1979), depresión (De Lange et al. 2002; Wall et al. 1996; Karasek 1979); ansiedad (Griffin et al. 2007; Wall et al. 1996); percepción de estrés (Fillion et al. 2007; Martín et al. 2007; Mikkelsen, Ǿgaard, Landsbergis 2005; Vermeulen y Mustard 2000); percepción de salud (Mikkelsen, Ǿgaard, Landsbergis 2005); accidentes laborales y bajas por enfermedad (Martín et al. 2007).

Generalmente, los problemas de salud laboral que se han tratado de explicar con el Modelo JDC(-S) se han abordado con la óptica de la presencia real del problema, tanto desde un punto de vista objetivo - basado en diagnósticos técnicos-, como subjetivo -basado en la percepción o autodiagnóstico de los trabajadores-. Sin embargo, es menos habitual el enfoque de la salud laboral desde el punto de vista de la potencialidad percibida por los trabajadores del riesgo de llegar a sufrir una enfermedad o accidente. Es precisamente sobre este punto, menos habitual en los estudios sobre el estrés, y sin embargo de gran relevancia como se verá más abajo, sobre el que se ha enfocado la presente investigación.

La concepción subjetiva del riesgo ha ido cobrando importancia en el ámbito de la investigación a través del concepto "riesgo percibido" (Sjöberg 2000; 2002), el cual engloba aspectos tanto situacionales, como físicos o personales. En el ámbito laboral se postula que el riesgo percibido condiciona la reacción o conducta preventiva del trabajador (Fischhoff et al. 1981, cit. en Zimolong y Trimpop 1998; Portell et al. 1997). Si bien es preciso que exista un riesgo laboral -objetivo- para que se dé un accidente, no puede subestimarse el riesgo subjetivo, ya que en muchas ocasiones el trabajador detecta la existencia de riesgos reales que, objetiva o científicamente, no se han podido determinar. En suma, si el trabajador percibe de manera subjetiva la existencia de un riesgo, aunque de manera objetiva no exista, el trabajador se comportará como si realmente existiese (García 2006).

El concepto de riesgo tiene la doble acepción de "peligro" y "oportunidad". Los estudios de seguridad han reducido en gran medida el significado de riesgo a sus perfiles de peligro y solo últimamente se han revalorizado sus consecuencias positivas - por ejemplo la adopción de una conducta más prudente. Con este punto de vista, sería deseable que el trabajador percibiera los riesgos asociados a su trabajo para que internalizara 
comportamientos más seguros. Sin embargo, nos encontramos con un problema cuando se da una percepción distorsionada del riesgo de padecer una enfermedad o de sufrir un accidente. Ya que, si es exagerada, puede acarrear que el trabajador manifieste un exceso de prudencia; por el contrario, una percepción devaluativa del riesgo puede fomentar un comportamiento imprudente. En este sentido, entender cómo perciben los trabajadores el riesgo de tener un accidente $o$ una enfermedad laboral es un asunto destacable para el diseño de políticas organizativas que traten de garantizar una conducta segura (Alonso y Pozo 2011).

Cabe entonces cuestionarse si las características del trabajo generadoras 0 atenuadoras de tensión mental, postuladas por el Modelo JDC(-S), influyen en la percepción del riesgo de accidente o enfermedad. De ser así, la política de rediseño organizativo tiene otra perspectiva interesante de trabajo, por cuanto puede coadyuvar, no solo a disminuir el estrés nocivo - y en consecuencia prevenir otro tipo de problemas de salud relacionados-, sino también a un comportamiento más seguro del trabajador, evitando percepciones distorsionadas del riesgo de salud.

El principal objetivo de esta investigación es estudiar de qué modo los componentes del modelo JDC(-S) influyen en el riesgo percibido de enfermedad o accidente en el lugar de trabajo y, en concreto, analizar el papel del Control y del Apoyo como variables moduladoras entre las Demandas y el Riesgo percibido. La novedad de este trabajo de investigación reside precisamente en la consideración de los componentes del Modelo JDC(-S) a efectos de explicar la percepción de Riesgo subjetivo. Es decir, se pretende evaluar si el trabajador percibe como riesgo sobre su salud lo que numerosos estudios vienen demostrando sobre el impacto del estrés laboral en la salud física y psíquica.

La medición de los componentes del modelo JDC(-S) y del Riesgo percibido se operacionaliza a partir de la Primera Encuesta Andaluza de Condiciones de Trabajo realizada por el Instituto Andaluz de Prevención de Riesgos Laborales, de la Consejería de Empleo de la Junta de Andalucía'. La utilización de dicha base de datos tiene fortalezas, pero también debilidades. El principal punto fuerte es la posibilidad de disponer de más de 7500 registros completos; aunque, como contrapartida, presenta una limitación destacable en la adecuación muestral de los indicadores de las escalas, validez de contenido, en cuanto a muestras de un dominio más amplio de indicadores representativos de los componentes del modelo JDC(-S) y del Riesgo percibido. Por ello, adicionalmente al objetivo descrito, este trabajo valorará la adecuación de la estructura dimensional del modelo JDC(-S), dados los indicadores seleccionados con tal fin de medición y el instrumento utilizado. Así mismo, cabe mencionar el uso, como herramienta de análisis, de los Modelos de Ecuaciones Estructurales con Variables Latentes (MEE-VL), también denominados Modelos de Estructuras de Covarianza, guiándose su aplicación por los principios de la regresión jerárquica (Bentler y Satorra 2000; Cohen y Cohen 1983), esto

\footnotetext{
${ }^{1}$ Se agradece a la Consejería de Empleo de la Junta de Andalucía la cesión gratuita de la Base de datos de la Primera Escuela Andaluza de condiciones de Trabajo 2008.
} 
es, las variables explicativas son introducidas en la ecuación de regresión en el orden sugerido por las hipótesis planteadas. Esta aproximación, entre otras ventajas, permite al investigador, cuando el número de indicadores por variable latente no es muy numeroso, estimar las variables latentes, esto es, tener en cuenta el error de medición de las variables observadas.

El artículo se estructura de la siguiente manera: Primero, se desarrolla el marco teórico sobre el que se justifican las hipótesis del estudio, resaltando la polémica existente en torno a la dimensionalidad de las escalas del componente Demandas y la naturaleza de las relaciones (directas versus moduladoras) entre las variables. Seguidamente, se expone el método de trabajo, en términos de descripción de la muestra, del instrumento y del procedimiento utilizado, así mismo se detallan los análisis. Tras dicha exposición se da cuenta de los principales resultados. Finalmente, se concluye y se presentan las principales limitaciones del estudio.

\section{MARCO TEÓRICO}

El Modelo JDC planteaba que la Tensión Mental en el trabajo y sus consecuencias físicas y psicológicas resultan de la interacción entre las Demandas del trabajo y el Control que se ejerza sobre este. Las Demandas del trabajo se definían como: sobrecarga mental, restricciones organizacionales para realizar las tareas, o demandas conflictivas; mientras que el Control sobre el trabajo hacía referencia a la posibilidad de tomar decisiones y usar las propias competencias y capacidades (Karasek 1979). El modelo proponía que altas Demandas actúan como estresores elicitando reacciones de estrés, y que los efectos de la Tensión mental más adversos para la salud se daban en situaciones de altas Demandas y bajo Control. Esta combinación de características del trabajo es lo que se denominaba "trabajo altamente estresante".

En los años 80 el modelo fue desarrollado, siendo la extensión más importante la incorporación de una tercera dimensión: el Apoyo social en el lugar de trabajo (Johnson y Hall 1988). Este nuevo factor hace referencia tanto a la ayuda interna de compañeros de trabajo y jefes, como a la externa. De acuerdo con este modelo extendido: Demandas-Control-Apoyo, Modelo JDC-(S), el mayor riesgo de problemas físicos y psicológicos se daría en grupos aislados, con trabajos caracterizados por altas Demandas, bajo Control y bajo Apoyo social (Karasek y Theorell 1990). El modelo proponía que el Apoyo mitiga el impacto negativo de la alta Tensión mental (altas Demandas-bajo Control). Johnson y Hall predecían que incrementos del Control amortiguaban las altas Demandas de forma más efectiva cuando se daban bajo condiciones de alto Apoyo social. En una revisión compiladora de la literatura sobre el modelo JDC y JDC(-S) en el periodo 1998-2009, Häusser (2010) muestra que el Modelo JDC(-S) ha obtenido unos ratios de confirmación más reducidos que el Modelo JDC, lo que indicaría problemas asociados con la dimensión del Apoyo social. Si bien, según el propio Häusser, estos problemas 
pueden deberse a meros efectos estocásticos asociados a la inclusión en el modelo de una tercera variable. Otra posible explicación podría ser que, tal y como sugieren otras investigaciones, solo en los casos de muy baja presencia de Apoyo social se observa un decrecimiento en el nivel de bienestar físico y/o mental. Es decir, que una vez que cierto nivel de Apoyo social es alcanzado, un incremento subsecuente no resulta en un aumento comparable de bienestar (Castro, Campero y Hernández 1997).

A pesar de las numerosas evidencias empíricas convincentes, ha habido una crítica sustancial acerca de la dimensionalidad del Modelo JDC(-S) y de los instrumentos utilizados para medirlo (Mikkelsen, Ǿgaard, Landsbergis 2005). Las principales críticas se han dirigido hacia la dimensionalidad de las escalas del factor Demandas y hacia la consistencia de las interacciones entre variables. A continuación se abordan estos dos aspectos por separado.

En el modelo original de Karasek, la escala de Demandas del trabajo contenía principalmente medidas de sobrecarga, lo que se ajustaba a las características de una sociedad industrial donde la sobrecarga era el estresor más relevante (Mikkelsen, Ǿgaard, Landsbergis 2005). Aunque se reconocía que podía haber otros tipos de Demandas, no era habitual su modelización. Karasek y Theorell, por ejemplo, discutieron la dificultad de conceptualizar y medir Demandas debido a la diversidad de subcomponentes. Sin embargo, los requerimientos de la sociedad postindustrial han hecho que vuelva a reconsiderarse esta cuestión. Por ejemplo: las tecnologías de la información han incrementado las exigencias cognitivas con vistas a la toma de decisiones, a un conocimiento más profundo de las organizaciones complejas y a la habilidad para analizar y resolver problemas en situaciones de incertidumbre (Mikkelsen, Ǿgaard, Landsbergis 2005).

Así, frente a la unidimensionalidad global del constructo Demandas que se ha empleado en muchas investigaciones (Tucker et al. 2008; Grönlund 2007; Martín, Salanova y Peiró 2007; Sanne et al. 2005; Schaufeli y Bakker 2004; Bakker, Demerouti y Verbeke 2004; Pelfrene et al. 2001; Rafferty, Friend y Landsbergis 2001; Janssen 2000; Sargent y Terry 2000; Wall et al. 1996; Karasek 1979), en otras, se ha buscado una mayor capacidad explicativa diferenciando entre una dimensión "cuantitativa o sobrecarga" - relacionada con ritmos y plazos_, y una dimensión "cualitativa" —relacionada con exigencias cognitivas, sensoriales o emocionales- (Martín et al. 2007; Mikkelsen, Ǿgaard, Landsbergis 2005; Kristensen y Borg 2001; Wall, Jackson y Mullarkey 1995).

En esta segunda línea se han obtenido evidencias de mejores ajustes del modelo JDC(-S). Por ejemplo, Mikkelsen, Ǿgaard, Landsbergis (2005) encuentran que, al diferenciar entre Demandas, mejora la varianza explicada con relación al estrés laboral $(26 \%)$, a la satisfacción en el trabajo (20\%) o, en menor medida, a otras variables como "quejas de salud subjetivas" (10\%). Al mismo tiempo, encontraron que diferentes tipos de Demandas eran predictivos con relación a ciertas variables y a otras no. Así, por ejemplo, las Demandas cognitivas eran asociadas con el estrés, pero no con el aprendizaje. En este mismo sentido, Martín et al. (2007) encuentran una asociación de Demandas cognitivas con el estrés o la fatiga, pero no con la satisfacción o los accidentes laborales. 
La bidimensionalidad del constructo Demandas puede llevar también aparejada un peso diferente de cada dimensión sobre la variable resultado. Este aspecto tiene importantes consecuencias teóricas y prácticas. De un lado, mezclar diferentes tipos de Demandas en una única dimensión puede hacer que los efectos de unas se compensen con los de otras y se pierda riqueza informativa. De otro, si se obtuvieran distintos efectos, la diferenciación dimensional puede conducir a una política más efectiva de rediseño del puesto de trabajo.

Las evidencias confirmatorias del Modelo JDC(-S), sea conceptualizando una o varias dimensiones de Demanda, han venido a fortalecer la asociación positiva entre Demandas y problemas o enfermedades tanto físicos como psicológicos, así como los efectos negativos del Control y del Apoyo sobre estos. De ahí que en el caso de la variable de estudio que nos ocupa: el Riesgo percibido de accidente o enfermedad, podría esperarse el mismo tipo de asociaciones.

Con relación a las interacciones entre variables -que uno de los componentes del modelo pueda influir o modificar el efecto sobre la "variable resultado" de los otros componentes-, estas pueden adoptar varias formas. En concreto, respecto a la relación entre Demandas y Control (y Apoyo) y la Tensión mental y sus consecuencias físicas y psicológicas sobre los trabajadores se han postulado generalmente dos tipos de efectos: aditivos (additive effect) y multiplicativos (multiplicative interaction) ${ }^{2}$ (Van Vegchel, Jan de Jonge y Landsbergis 2005:538). Mientras Karasek puso poco énfasis en la distinción entre estos dos tipos de efectos (Häusser et al. 2010), otros investigadores han señalado que esta distinción es de gran relevancia para la aceptación del Modelo JDC (-S). La confirmación de uno u otro tipo de efecto tendría distintas implicaciones tanto para el diseño del lugar del trabajo, como para las intervenciones organizacionales que buscaran prevenir la Tensión mental entre los empleados. Así, si el efecto modulador es válido, habría que incrementar el Control (-Apoyo) para contrarrestar la alta Tensión mental; mientras que no sería necesario reducir las Demandas. Por el contrario, un aumento del Control (-Apoyo) no tendría el mismo efecto si estuviera conectado solo directamente con las Demandas. En este caso, la alta Tensión mental se podría reducir mediante medidas que mejoraran el Control (-Apoyo) pero los niveles de Tensión mental continuarían indeseablemente altos en tanto las Demandas permanecieran altas.

Tanto en la revisión compilatoria de la literatura realizada por Van der Doeff y Maes (1999) como en la de Häusser (2010), la existencia de efectos directos es un hecho bastante contrastado y poco discutido. Así, en el período 1998-2009, el 99\% de los

${ }^{2}$ Van Vegchel y colaboradores también se refieren a un tercer tipo de efecto: ratio o proporción, menos habitual. Los efectos aditivos son también denominados comúnmente efectos directos de la ecuación de regresión, mientras que los multiplicativos representarían los efectos moduladores (también denominados moderadores o interactivos). En esta investigación utilizaremos el término modulación (López-Arazo, Osca Segovia y Peiró 2007). 
estudios revisados confirmaban estos efectos total o parcialmente. Sin embargo, el estatus empírico relativo a los efectos moduladores es menos conclusivo. Así, solo el $30 \%$ de los estudios revisados en el período anterior apoyan la existencia de estos efectos parcial o totalmente. Si bien, los resultados encontrados mejoran los del período anterior (1979-1997).

Ante la hipótesis que pudiera albergarse sobre la inexistencia de efectos moduladores, el propio Häusser argumenta en contra en virtud de las evidencias obtenidas al analizar los diferentes trabajos revisados. Según este autor, no puede concluirse con una hipótesis nula dado que la influencia del Control y del Apoyo sobre las Demandas difiere según cómo se operacionalicen estos constructos, y que a la luz de las evidencias encontradas por los autores hay que descartar errores tipo I y efectos distorsionantes debidos a los tamaños muestrales. El problema no es tanto si existen estos efectos cuanto la manera de medirlos. Así por ejemplo, en el caso del Apoyo social algunos autores han señalado como problemas en su operacionalización y medida los siguientes. Por un lado, aun existiendo sobre el apoyo un concepto genérico común, este puede entenderse y abordarse desde perspectivas diferentes y no excluyentes. Algunos de estos problemas tienen que ver con la elección de medidas objetivas o subjetivas, la orientación de las medidas (centradas en el emisor, en el receptor o en ambos), tipo de medidas (apoyo general o centrado en problemas específicos), uso de medidas estructurales o funcionales, fuentes de apoyo, contenido del apoyo (emocional, instrumental, informativo o evaluativo), enfoque descriptivo o evaluativo y disponibilidad del apoyo (Pérez y Martín 1997).

Hipótesis 1. La componente de Demandas del trabajo o estresores en el trabajo consta de dos dimensiones relacionadas pero independientes -Demandas cuantitativas y Demandas cualitativas.

Hipótesis 2. Las Demandas cuantitativas-cualitativas, influyen positivamente en el Riesgo percibido de sufrir un accidente-enfermedad, mientras que el Control y el Apoyo influyen negativamente.

Paralelamente a la hipótesis dos, se formula una tercera hipótesis de trabajo. Más concretamente, esta hipótesis sintetiza dos posibles modulaciones, esto es, por un lado del Control y por el otro del Apoyo, en el efecto directo de las Demandas del trabajo, 0 estresores, sobre el Riesgo percibido.

Hipótesis 3a: El efecto de las Demandas sobre el Riesgo percibido se reduce conforme aumenta el grado de Control.

Hipótesis 3b: El efecto de las Demandas sobre el Riesgo percibido se reduce conforme aumenta el grado de Apoyo 


\section{MÉTOdo}

En este estudio se hace uso de la base de datos generada por la I Encuesta Andaluza de Condiciones de Trabajo, realizada en 2008 por el Instituto Andaluz de Prevención de Riesgos Laborales, de la Consejería de Empleo de la Junta de Andalucía, y que fue cedida a los autores para su explotación con fines de investigación.

\section{Muestra}

La muestra empleada en este estudio no fue obtenida ad hoc, sino que procede, como se ha dicho, de la I Encuesta Andaluza de Condiciones de Trabajo. Aunque los autores no han participado directamente en el diseño de la muestra si queremos poner de manifiesto la relevancia de estos datos aportando alguna información sobre su obtención y composición (Cuadro 1).

Respecto a la recogida de información, según el informe de la Primera Encuesta Andaluza de Condiciones de Trabajo, esta se llevó a cabo mediante entrevistas personales realizadas en el domicilio de los entrevistados, seleccionados mediante el método de rutas aleatorias. La elección de la última unidad de investigación: el individuo dentro del hogar, se hizo igualmente mediante un cuadro de decisión aleatorio y según cuotas establecidas.

La muestra obtenida fue de 8275 entrevistas, realizadas en 139 municipios andaluces. Por provincias, el $8,6 \%$ de la muestra corresponde a Almería, el $14,9 \%$ a Cádiz, el $9,8 \%$ a Córdoba, el 9,9\% a Granada, el 6,8\% a Huelva, el 7,5\% a Jaén, el $17,9 \%$ a Málaga y el $24,5 \%$ a Sevilla. El tratamiento de los datos ausentes seleccionado para el conjunto de variables observadas fue el listwise delection, lo que condujo a una matriz informativa final de 7512 registros, frente a los 8275 iniciales (esto representa aproxima-

\section{Cuadro 1.}

\section{Ficha técnica del estudio}

\begin{tabular}{|c|c|c|}
\hline Ámbito geográfico & \multicolumn{2}{|c|}{ Comunidad Autónoma andaluza. } \\
\hline Recogida de información & \multicolumn{2}{|c|}{ Entrevista personal en el domicilio, desde el 21 de enero al 30 de abril de 2008.} \\
\hline Universo de análisis & \multicolumn{2}{|c|}{ Población trabajadora residente en municipios de más de 5000 habitantes. } \\
\hline Tamaño de la muestra & \multicolumn{2}{|c|}{8275 entrevistas } \\
\hline Error muestral & \multicolumn{2}{|c|}{$\begin{array}{l}\text { El margen de error para el total de la muestra es de } \pm 1,1 \% \text { para un margen de } \\
\text { confianza del } 95 \% \text { y bajo el supuesto de máxima indeterminación }(p=q=50 \%) \text {. }\end{array}$} \\
\hline \multirow{4}{*}{$\begin{array}{l}\text { Procedimiento de muestreo } \\
\text { estratificado polietápico } \\
\text { aleatorio: }\end{array}$} & \multirow{2}{*}{ Cálculo } & Primer Estrato: PROVINCIAS Y RAMA DE ACTIVIDAD \\
\hline & & Segundo Estrato: SEXO Y TAMAÑO DE LA EMPRESA \\
\hline & \multirow[b]{2}{*}{ Unidades } & Primarias de muestreo (HOGARES) método de rutas aleatorias \\
\hline & & $\begin{array}{l}\text { Secundarias (INDIVIDUOS) mediante cuadro de decisión } \\
\text { aleatorio. }\end{array}$ \\
\hline
\end{tabular}

Fuente: Elaboración propia, a partir de la Primera Encuesta Andaluza de Condiciones de Trabajo del 2008. 
damente el $91 \%$ de los casos con respuestas válidas relativos al conjunto de variables objeto de análisis en este trabajo). Una descripción de los registros finales se muestra en la Tabla 1. El 66,4\% de la muestra son hombres y el 33,6\% mujeres. La edad media es aproximadamente de 36 años, siendo las mujeres más jóvenes que los hombres, por término medio. Respecto a la edad y los sectores, destaca que los encuestados que desarrollan su actividad tanto en el sector construcción como en el sector servicios son relativamente más jóvenes que los que trabajan en el sector industria y/o agropecuario. Estas últimas diferencias son significativas tal y como muestra el ANOVA realizado. Respecto a la potencial relación existente entre Sexo y Sector, tras obtener el estadístico chi-cuadrado y sus correspondientes residuos corregidos, se observan diferencias significativas entre el porcentaje de hombres y mujeres en los sectores de industria y construcción; especialmente en este último, donde el porcentaje de mujeres apenas sobrepasa el 6\%. El mayor porcentaje de mujeres se encuentra en el sector servicios.

Tabla 1.

Características de la muestra

\begin{tabular}{|c|c|c|c|c|c|c|c|c|c|c|}
\hline & & \multicolumn{3}{|c|}{ EDAD } & \multicolumn{2}{|c|}{ SEXO } & \multicolumn{4}{|c|}{ SECTOR } \\
\hline & & $\bar{X}$ & DT. & & $\mathrm{H}$. & M. & I. & C. & S. & A. \\
\hline & MUESTRA (N: 7.512) & 35,53 & 10,30 & $\% \mathrm{C}$ & $\% \mathrm{C}$ & $\% \mathrm{C}$ & $\% \mathrm{C}$ & $\% \mathrm{C}$ & $\% \mathrm{C}$ & $\% \mathrm{C}$ \\
\hline \multirow[t]{3}{*}{ SEXO } & H. Hombres (N: 4.990) & 36,49 & 10,50 & 66,4 & & & 78,1 & 93,9 & 54,6 & 67,9 \\
\hline & M. Mujeres (N: 2.522) & 33,63 & 9,67 & 33,6 & & & 21,9 & 6,1 & 45,4 & 32,1 \\
\hline & & & & & & & \multicolumn{4}{|c|}{ Chi-cuadrado: $675,73^{* *}$} \\
\hline \multirow[t]{4}{*}{ SECTOR } & I. Industria (N: 1.812) & 36,79 & 10,46 & 24,1 & 28,4 & 15,7 & & & & \\
\hline & C. Construcción (N: 918) & 34,06 & 10,02 & 12,2 & 17,3 & 2,2 & & & & \\
\hline & S. Servicios (N: 4.021) & 35,18 & 10,22 & 53,5 & 44,0 & 72,4 & & & & \\
\hline & A. Agricultura (N: 761) & 36,19 & 10,30 & 10,1 & 10,3 & 9,7 & & & & \\
\hline EDAD & & & & & \multicolumn{2}{|c|}{ t-test: $131,83^{* *}$} & \multicolumn{4}{|c|}{ ANOVA: $17,98^{* *}$} \\
\hline
\end{tabular}

$\%$ C: Porcentaje sobre columna.

Chi-cuadrado: Estadístico Chi-cuadrado para valorar la relación entre sexo y sector.

$t$ : Prueba t para igualdad de medias de edad respecto a sexo.

ANOVA: Análisis de la varianza, estadístico $\mathrm{F}$ para contrastar la igualdad conjunta de medias.

${ }^{* *}$ El estadístico es significativo al nivel 0,01.

\section{Indicadores}

Para la operacionalización de las variables objeto de estudio se partió de la revisión de las diferentes propuestas existentes en la literatura, y tomando como base la Encuesta Andaluza de Condiciones de Trabajo, se seleccionaron doce indicadores. En el Cuadro 2 se presenta un resumen que relaciona dichos indicadores con las variables objeto de estudio y autores que los utilizaron en sus trabajos. 


\section{Cuadro 2.}

Indicadores propuestos

\begin{tabular}{|c|c|c|}
\hline \multicolumn{2}{|l|}{ DEMANDAS CUANTITATIVAS } & $\begin{array}{l}\text { Formulación pregunta en } \\
\text { la I Encuesta Andaluza de } \\
\text { Condiciones de Trabajo }\end{array}$ \\
\hline X1: Trabajar muy rápido & $\begin{array}{l}\text { Karasek, 1979; Pelfrene et al. 2001; Janssen, } \\
\text { 2000; Schaufeli and Bakker, 2004; Bakker, Deme- } \\
\text { routi and Verbeke, 2004; MacDonald, Karasek, } \\
\text { Punnett and Scharf, 2001; Job Stress Question- } \\
\text { naire; ISTAS21; Job Content Questionnaire }\end{array}$ & $\begin{array}{l}\text { ¿En qué medida su trabajo } \\
\text { implica trabajar muy rápido? } \\
\text { (Escala } 1 \text { a } 5 / \text { Siempre o casi } \\
\text { siempre a Casi nunca o nunca) }\end{array}$ \\
\hline X2: Plazos estrictos-cortos & $\begin{array}{l}\text { Karasek, 1979; Pelfrene et al. 2001; Janssen, } \\
\text { 2000; Schaufeli and Bakker, 2004; MacDonal et } \\
\text { al. 2001; Cabezas, 2000; Job Stress Question- } \\
\text { naire; ISTAS21; Job Content Questionnaire }\end{array}$ & $\begin{array}{l}\text { ¿En qué medida su trabajo } \\
\text { implica trabajar con plazos } \\
\text { muy estrictos y muy cortos? } \\
\text { (Escala } 1 \text { a } 5 / \text { Siempre o casi } \\
\text { siempre a Casi nunca o nunca) }\end{array}$ \\
\hline \multicolumn{3}{|l|}{ DEMANDAS CUALITATIVAS } \\
\hline $\begin{array}{l}\text { X3: Trabajo intelectualmente } \\
\text { exigente }\end{array}$ & $\begin{array}{l}\text { Wall, Jackson and Mullarkey, 1995; Karasek, } \\
\text { 1990; Martín, Luceño, Jaen y Rubio, 2007; Cabe- } \\
\text { zas, 2000; ISTAS21 }\end{array}$ & $\begin{array}{l}\text { Considera que su trabajo es } \\
\text { intelectualmente exigente } \\
\text { (Escala } 1 \text { a } 5 / \text { Siempre o casi } \\
\text { siempre a Casi nunca o nunca) }\end{array}$ \\
\hline $\begin{array}{l}\text { X4: Trabajo emocionalmente } \\
\text { exigente }\end{array}$ & $\begin{array}{l}\text { Wall, Jackson and Mullarkey, 1995; Schaufeli and } \\
\text { Bakker, 2004; Bakker, Demerouti and Verbeke, } \\
\text { 2004; Karasek, 1990; Cabezas, 2000; ISTAS21 }\end{array}$ & $\begin{array}{l}\text { Considera que su trabajo es } \\
\text { emocionalmente exigente } \\
\text { (Escala } 1 \text { a } 5 / \text { Siempre o casi } \\
\text { siempre a Casi nunca o nunca) }\end{array}$ \\
\hline \multicolumn{3}{|l|}{ CONTROL } \\
\hline $\begin{array}{l}\text { X5: Poder elegir o modificar } \\
\text { el orden de las tareas }\end{array}$ & $\begin{array}{l}\text { Karasek, 1979; Martín, Salanova y Peiró, 2007; } \\
\text { Sargent y Terry, 2000; Wall et al. 1996; Pelfrene } \\
\text { et al. 2001; Wall, Jackson and Mullarkey, 1995; } \\
\text { Wong, DeSantics Staudemayer, 2007; Grönlund, } \\
\text { 2007; Bakker, Demerouti and Verbeke, 2004; } \\
\text { Karasek, 1990; Martín et al. 2007; MacDonald et } \\
\text { al. 2001; ISTAS21; Job Content Questionnaire. }\end{array}$ & $\begin{array}{l}\text { ¿Puede elegir o modificar el } \\
\text { orden de las tareas? } \\
\text { (Escala } 1 \text { a } 5 / \\
\text { Sí, siempre que quiero a Casi } \\
\text { nunca o nunca) }\end{array}$ \\
\hline $\begin{array}{l}\text { X6: Poder elegir o modificar } \\
\text { el método de trabajo }\end{array}$ & $\begin{array}{l}\text { Karasek, 1979; Martín, Salanova y Peiró, 2007; } \\
\text { Sargent y Terry, 2000; Wall et al. 1996; Pelfrene } \\
\text { et al. 2001; Wall, Jackson and Mullarkey, 1995; } \\
\text { Wong DeSantics Staudemayer, 2007; Grönlund, } \\
\text { 2007; Bakker, Demerouti and Verbeke, 2004; } \\
\text { Karasek, 1990; Martín et al. 2007; MacDonald et } \\
\text { al. 2001; ISTAS21; Job Content Questionnaire. }\end{array}$ & $\begin{array}{l}\text { ¿Puede elegir o modificar el } \\
\text { método de trabajo? } \\
\text { (Escala } 1 \text { a } 5 / \text { Sí, siempre que } \\
\text { quiero a Casi nunca o nunca) }\end{array}$ \\
\hline $\begin{array}{l}\text { X7: Poder elegir o modificar } \\
\text { el ritmo de trabajo }\end{array}$ & $\begin{array}{l}\text { Karasek, 1979; Martín, Salanova y Peiró, 2007; } \\
\text { Sargent y Terry, 2000; Wall et al. 1996; Pelfrene } \\
\text { et al. 2001; Wall, Jackson and Mullarkey, 1995; } \\
\text { Wong DeSantics Staudemayer, 2007; Grönlund, } \\
\text { 2007; Bakker, Demerouti and Verbeke, 2004; } \\
\text { Karasek, 1990; Martín et al. 2007; MacDonald et } \\
\text { al. 2001; ISTAS21; Job Content Questionnaire. }\end{array}$ & $\begin{array}{l}\text { ¿Puede elegir o modificar el } \\
\text { ritmo de trabajo? } \\
\text { (Escala } 1 \text { a } 5 / \text { Sí, siempre que } \\
\text { quiero a Casi nunca o nunca) }\end{array}$ \\
\hline
\end{tabular}




\section{Cuadro 2.}

Continuación

\begin{tabular}{|c|c|c|}
\hline \multicolumn{2}{|l|}{ DEMANDAS CUANTITATIVAS } & $\begin{array}{c}\text { Formulación pregunta en } \\
\text { la I Encuesta Andaluza de } \\
\text { Condiciones de Trabajo }\end{array}$ \\
\hline $\begin{array}{l}\text { X8: Poder elegir o modificar } \\
\text { la distribución/ } \\
\text { duración pausas }\end{array}$ & $\begin{array}{l}\text { Karasek, 1979; Martín, Salanova y Peiró, 2007; } \\
\text { Sargent y Terry, 2000; Wall et al. 1996; Pelfrene } \\
\text { et al. 2001; Wall, Jackson and Mullarkey, 1995; } \\
\text { Wong DeSantics Staudemayer, 2007; Grönlund, } \\
\text { 2007; Bakker, Demerouti and Verbeke, 2004; } \\
\text { Karasek, 1990; Martín et al. 2007; MacDonald et } \\
\text { al. 2001; ISTAS21; Job Content Questionnaire. }\end{array}$ & $\begin{array}{l}\text { ¿Puede elegir o modificar la } \\
\text { distribución y/o duración de las } \\
\text { pausas en el trabajo? } \\
\text { (Escala } 1 \text { a } 5 / \text { Sí, siempre que } \\
\text { quiero a Casi nunca o nunca) }\end{array}$ \\
\hline \multicolumn{3}{|l|}{ APOYO } \\
\hline X9: Ayuda de compañeros & $\begin{array}{l}\text { Sargent y Terry, 2000; Pelfrene et al. 2001; } \\
\text { Schaufeli and Bakker, 2004; Bakker, Deme- } \\
\text { routi and Verbeke, 2004; Martín et al. 2007; } \\
\text { MacDonald et al. 2001; Cabezas, 2000; Job } \\
\text { Stress Questionnaire; ISTAS21; Job Content } \\
\text { Questionnaire. }\end{array}$ & $\begin{array}{l}\text { Puede obtener ayuda de sus } \\
\text { compañeros si la pide } \\
\text { (Escala } 1 \text { a } 5 / \text { Siempre o casi } \\
\text { siempre a Casi nunca o nunca) }\end{array}$ \\
\hline X10: Ayuda de superiores & $\begin{array}{l}\text { Sargent y Terry, 2000; Pelfrene et al. 2001; } \\
\text { Schaufeli and Bakker, 2004; Martín et al. 2007; } \\
\text { MacDonald et al. 2001; Cabezas, 2000; Job } \\
\text { Stress Questionnaire; ISTAS21; Job Content } \\
\text { Questionnaire. }\end{array}$ & $\begin{array}{l}\text { Puede obtener ayuda de sus } \\
\text { superiores/jefes si la pide } \\
\text { (Escala } 1 \text { a } 5 / \text { Siempre o casi } \\
\text { siempre a Casi nunca o nunca) }\end{array}$ \\
\hline \multicolumn{3}{|l|}{ RIESGO PERCIBIDO } \\
\hline $\begin{array}{l}\text { Y1: Riesgo de sufrir una } \\
\text { enfermedad }\end{array}$ & $\begin{array}{l}\text { Kleinman, Eisenberg y Good,1978; Karasek, 90; } \\
\text { MacDonald et al. 2001; Martín et al. } 2007 .\end{array}$ & $\begin{array}{l}\text { Hasta que punto le preocupa } \\
\text { de su trabajo el riesgo de sufrir } \\
\text { una enfermedad } \\
\text { (Escala } 1 \text { a 5/Nada a Mucho) }\end{array}$ \\
\hline $\begin{array}{l}\text { Y2: Riesgo de tener un } \\
\text { accidente }\end{array}$ & $\begin{array}{l}\text { Kleinman, Eisenberg y Good,1978; Quick, Quick, } \\
\text { Nelson y Hurrell, 1997; Martín et al. } 2007 .\end{array}$ & $\begin{array}{l}\text { Hasta que punto le preocupa } \\
\text { de su trabajo el riesgo de tener } \\
\text { un accidente } \\
\text { (Escala } 1 \text { a } 5 / \text { Nada a Mucho) }\end{array}$ \\
\hline
\end{tabular}

Fuente: Elaboración propia.

Las variables sexo, edad y sector de actividad se emplearon como variables de control debido a su relevancia ampliamente demostrada para explicar diferencias tanto en las variables criterio o dependientes como en las explicativas o independientes (Gracia et al. 2007; Grönlund 2007, Martín et al. 2007; Wong et al. 2007; Melia y Becerril 2007; Rafferty, Friend y Landsbergis 2001; MacDonalds et al. 2001; Pelfrene et al. 2001; Janssen 2000; Wall et al. 1996; Wall, Jackson y Mullarkey 1995; Karasek 1990; Landsbergis 1988). La variable edad está medida en años por lo que es considerada continua, mientras sexo y sector son variables categóricas. En el caso de la variable sexo, dicotómica, 
se asocia el valor 0 para los hombres y el 1 para las mujeres. La variable sector consta de cuatro categorías, por lo que se crean tres variables dicotómicas, de ceros y unos, asociadas a los sectores: construcción, agricultura y servicios, respectivamente, esto es, la categoría que se toma como umbral de referencia es el sector industria.

\section{Análisis}

A efectos de valorar la inclusión de las variables de control, se calculan sus correspondientes pruebas de asociación con los indicadores. La métrica de las variables condiciona la metodología concreta que se aplique en cada caso. Con la variable sexo, en primer lugar, se da cuenta del contraste de igualdad de varianzas ("prueba de Levene" para la igualdad de varianzas). Así, en caso de rechazo, igualdad de varianzas, se aplica la correspondiente corrección en la prueba t para el contraste de igualdad de medias. La variable sector consta de 4 categorías, por lo que se presenta el análisis de la varianza (ANOVA) para el contraste conjunto del vector de medias, y el "Test de Duncan" para los contrastes dos a dos. En el caso de la edad, las potenciales asociaciones se sintetizan mediante los coeficientes de correlación de Pearson.

La Hipótesis 1 se pone a prueba a través de la valoración de la estructura dimensional de los componentes del modelo JDC(-S), dados los indicadores seleccionados en esta investigación. En primer lugar, se da cuenta de la matriz de correlaciones de Pearson, para posteriormente realizar un Análisis de Componentes Principales sobre dicha matriz con rotación oblicua. Este análisis clásico y exploratorio se complementa con el cálculo del alpha de Cronbach en su versión estandarizada (Nunnally y Bernstein 1994). Tras obtener evidencia de una potencial estructura dimensional subyacente al conjunto de variables observadas, la metodología clave para el resto del trabajo se concreta en los Modelos de Ecuaciones Estructurales con Variables Latentes (MEE-VL). Los MEE-VL proporcionan una serie de ventajas con respecto a otras. Una de las más importantes es que permite al investigador introducir tanto información a priori como valorar su inclusión y/o reformular sus modelos, sobre la base de estadísticos e índices de bondad de ajuste. Otra de las ventajas asociadas a MEE-VL es la amplia variedad de métodos de estimación que están disponibles en los programas estadísticos que los soportan, LISREL (Jöreskog y Sörbom 1996), MPLUS (Muthén y Muthén 1998-2007) y EQS (Bentler 1989-2006). En esta investigación se estiman los modelos con EQS6.2 mediante Máxima Verosimilitud, y se establecen las correcciones propuestas por Satorra y Bentler (1994) tanto en las estimaciones de los errores estándares de los parámetros estimados como en los estadísticos de bondad de ajuste.

La inclusión en los Modelos Estructurales de efectos moduladores implica el cálculo de nuevas variables. En este trabajo el número de indicadores por variable latente oscila entre 2 y 4 , por lo que, se estimarán las puntuaciones factoriales de las variables latentes. Así, se estiman las variables latentes, las componentes del modelo, para posteriormente calcular las variables representativas de los efectos moduladores, esto es, sus productos. La estimación de las puntuaciones factoriales se realiza 
mediante la estimación de un Modelo de Análisis Factorial Confirmatorio utilizando el estimador de Mínimos Cuadrados Generalizados para las puntuaciones factoriales (Bentler 1989-2006).

En el caso de la Hipótesis 2 se especifica un modelo de regresión jerárquica con variables observadas y variables latentes; un Modelo de Ecuaciones Estructurales con Variables Latentes (Bentler y Satorra 2000). Más concretamente, en el primer paso se introducen las variables de control: edad, sexo y sector, y en el segundo paso, se añaden las variables latentes representativas de los componentes del modelo JDC(-S). La Hipótesis 3 (sub-hipótesis 3a y 3b) se pone a prueba mediante la inclusión de un tercer paso, esto es, se añaden en la ecuación de regresión las variables representativas de los efectos moduladores. Así, dichas sub-hipótesis se ponen a prueba a partir del análisis de la significatividad del incremento de varianza explicada, para posteriormente analizar la significatividad individual de los parámetros y sus correspondientes rectas de regresión (Aiken y West 1991).

\section{Resultados}

En la Tabla 2 se sintetizan los estadísticos descriptivos y los resultados de los contrastes de igualdad de medias.

Como puede observarse, los indicadores de los componentes del modelo JDC(-S) que presentan las puntuaciones más altas son los relativos al Apoyo percibido, mientras que las más bajas corresponden al Control. Entre ambas se encuentran las valoraciones medias de los indicadores de Demanda. Si atendemos a las diferencias significativas de medias según las diferentes variables de control, cabe subrayar que la percepción de Demandas cuantitativas es superior en los hombres, así como en el sector de la construcción. Sin embargo, en el caso de las Demandas cualitativas son las mujeres las que perciben una mayor exigencia, siendo los sectores industrial y servicios donde se presentan unos valores de percepción más altos, así como entre los individuos de mayor edad. Según la variable sexo, el Control percibido es mayor en las mujeres para el caso de los indicadores "poder elegir o modificar el orden de las tareas" y "poder elegir o modificar el método de trabajo". Por sectores, el Control percibido es mayor en el sector servicios, al igual que lo que sucede para las edades superiores. Respecto al Apoyo, solo se han detectado diferencias, según la variable sexo, para el caso del indicador relativo al "Apoyo de los jefes", siendo las mujeres las que perciben un mayor nivel. Según sectores, los individuos del sector de la construcción son los que perciben un mayor apoyo de los compañeros, y los del sector agrario un mayor apoyo de los jefes. Por edades, ambos tipos de apoyo se perciben en mayor grado entre los individuos más jóvenes. Finalmente, el riesgo percibido es mayor entre los hombres, entre los trabajadores del sector de la construcción y entre los de mayor edad. 


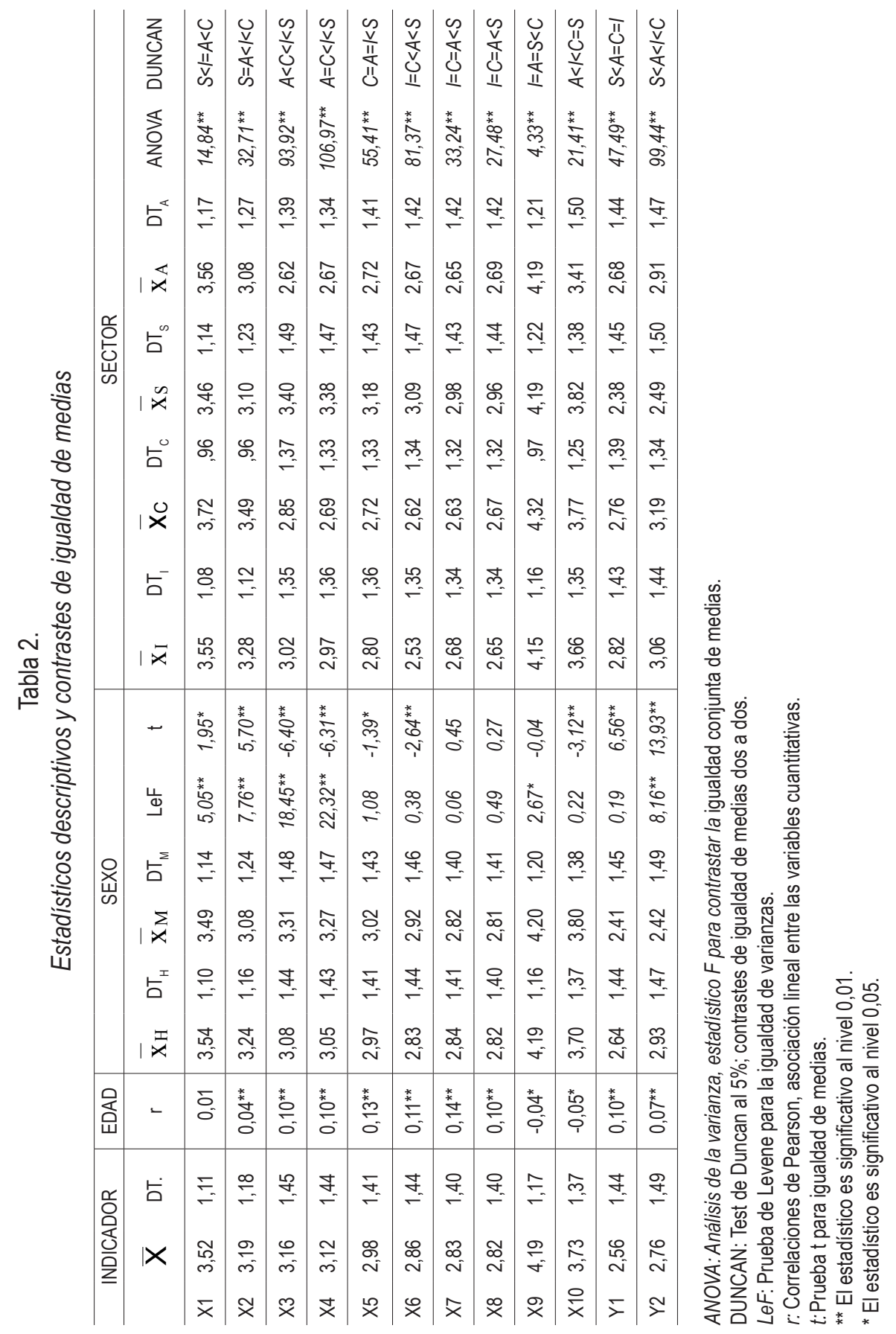


De la observación de la matriz de correlaciones entre los indicadores se deduce la existencia de una estructura dimensional subyacente y acorde al planteamiento formulado (Tabla 3). Las correlaciones más altas se detectan entre los indicadores asociados a una misma dimensión (entre los dos indicadores de Demanda cuantitativa: $r=0,62$; entre los dos indicadores de Demanda cualitativa: $r=0,68$; entre los cuatro indicadores de Control: $r=0,80-0,80-0,69-0,77-0,68-0,76$; entre los dos indicadores de Apoyo: $r=0,65$, entre los dos indicadores de Riesgo percibido: $r=0,77)$. El alpha de Cronbach ${ }^{3}$ para escala de Control alcanza el 0,92. Asimismo, las correlaciones existentes entre los indicadores de demanda cuantitativa versus cualitativa, aunque significativas, no son altas.

Tabla 3.

Matriz de correlaciones.

\begin{tabular}{|c|c|c|c|c|c|c|c|c|c|c|c|}
\hline & $\mathrm{X} 1$ & $\mathrm{X} 2$ & X3 & X4 & X5 & $\mathrm{X} 6$ & $\mathrm{X} 7$ & X8 & X9 & $\mathrm{X} 10$ & Y1 \\
\hline X2 & $0,62^{* *}$ & & & & & & & & & & \\
\hline X3 & $0,10^{* *}$ & $0,16^{* *}$ & & & & & & & & & \\
\hline X4 & $0,11^{* *}$ & $0,15^{\star *}$ & $0,68^{* *}$ & & & & & & & & \\
\hline X5 & $-0,02$ & $0,04^{* *}$ & $0,25^{\star *}$ & $0,26^{* *}$ & & & & & & & \\
\hline X6 & $-0,05^{\star \star}$ & 0,00 & $0,28^{\star *}$ & $0,26^{* *}$ & $0,80^{* *}$ & & & & & & \\
\hline $\mathrm{X} 7$ & $-0,04^{* *}$ & $-0,01$ & $0,28^{* *}$ & $0,25^{\star *}$ & $0,79^{* *}$ & $0,77^{* *}$ & & & & & \\
\hline X8 & $-0,04^{\star *}$ & $-0,01$ & $0,25^{\star *}$ & $0,21^{* *}$ & $0,69^{\star *}$ & $0,68^{* *}$ & $0,76^{\star *}$ & & & & \\
\hline $\mathrm{Xg}$ & $0,19^{* *}$ & $0,11^{\star *}$ & $0,12^{* *}$ & $0,08^{* *}$ & 0,01 & $0,01^{* *}$ & $0,04^{*}$ & $-0,00$ & & & \\
\hline $\mathrm{X} 10$ & $0,11^{\star *}$ & $0,06^{* *}$ & $0,14^{\star *}$ & $0,09^{* *}$ & 0,02 & $0,04^{\star *}$ & $0,05^{\star *}$ & $0,02^{*}$ & $0,65^{* *}$ & & \\
\hline Y1 & $0,15^{\star *}$ & $0,19^{\star \star}$ & 0,02 & $0,05^{* *}$ & $-0,08^{* *}$ & $-0,07^{* *}$ & $-0,06^{* *}$ & $-0,09^{* *}$ & $-0,05^{*}$ & $-0,08^{* *}$ & \\
\hline Y2 & $0,14^{* *}$ & $0,18^{\star *}$ & $-0,04^{* *}$ & 0,02 & $-0,05^{\star *}$ & $-0,03^{\star *}$ & $-0,02^{*}$ & $-0,05^{\star *}$ & $-0,00$ & $-0,06^{*}$ & $0,77^{* *}$ \\
\hline
\end{tabular}

* Significativa al nivel 0,05 (bilateral).

** Significativa al nivel 0,01 (bilateral).

Negritas: correlaciones entre los indicadores de una misma componente.

${ }^{3}$ En las escalas de dos ítem la correlación entre ellos es el alpha de Cronbach estandarizada. 
Los resultados más relevantes en términos medios del Análisis de Componentes Principales son los siguientes: los 4 primeros valores propios superan la unidad $(3,56-$ 2,01-1,42-1,22); el porcentaje de varianza explicada acumulada supera el $85 \%$. Las relaciones medias entre variables y componentes asignadas están en el rango 0,870,92 , por lo que sus correspondientes comunalidades superan el 0,75 , mientras que las relaciones variables-componentes no asignadas no alcanzan el 0,30. Así, en términos medios (extracciones muestrales aleatorias), los valores propios y los porcentajes de varianza explicada, así como, las comunalidades y cargas (tamaño y asociación), ponen de manifiesto la existencia de una estructura de cuatro componentes principales.

Tras estimar un Modelo de Análisis Factorial Confirmatorio con 4 variables latentes en nuestra matriz de datos (7.512 casos), la chi-cuadrado de Satorra-Bentler alcanza el valor de 478,15 con 29 grados de libertad, mientras que el R-RMSEA no supera el 0,045. Los índices GFI, AGFI, R-CFI superan en el 0,960 y el SRMR no alcanza el 0,030 por lo que existe evidencia para el no rechazo del modelo planteado (Hu y Bentler 1999; Hair et al. 2006).

Las estimaciones de los parámetros sugieren evidencia tanto de fiabilidad como de validez convergente (Tabla 4). Todas las cargas factoriales son significativas y están en el rango $0,68-0,90$, por lo que sus correspondientes coeficientes de varianza explicada oscilan entre 0,46 y 0,82 . Respecto a los coeficientes de Fornel y Larcker y omega de McDonald, se observa que sus valores mínimos alcanzan el 0,63 (CVE) y el 0,77 (CFC), respectivamente; superando los valores propuestos por Fornel y Larcker (1981) y remarcados por Bagozzi (2010). Paralelamente, todas las cargas factoriales superan a las correlaciones entre las dimensiones (la correlación más alta alcanza el 0,37 mientras que la carga más baja supera el 0,68 ), por lo que existe evidencia de validez discriminante (Bagozzi 2010; Anderson y Gerbing 1988). Asimismo, se observa que la dimensión Demandas cualitativas presenta relaciones significativas positivas tanto con el Control como con el Apoyo $(0,37$ y 0,14$)$, mientras que respecto a la Demanda cuantitativa destaca la correlación positiva con el Apoyo $(0,21)$. Las dimensiones Control y Apoyo no presentan una relación significativa. La validez predictiva y nomológica de las variables latentes se juzga en el marco teórico de los modelos planteados. El Modelo JDC(-S), en la muestra analizada y dada la operacionalización realizada de los indicadores y sus componentes, consta de cuatro dimensiones: Demandas Cuantitativas, Demandas Cualitativas, Control y Apoyo. Por lo que existe evidencia para no rechazar la primera hipótesis planteada, dos dimensiones de Demanda independientes pero relacionadas (su correlación es positiva y significativa: 0,17 ).

Los resultados de los tres modelos representativos de nuestras dos últimas hipótesis se presentan en el Tabla 5. Respecto a los índices de bondad de ajuste global de los tres modelos, en primer lugar, señalar que los tres estadísticos chi-cuadrado de SatorraBentler alcanzan valores significativos, sin embargo, el R-RMSEA no supera el 0,06. Además, los índices de ajuste GFI, AGFI, R-CFI superan en todos los casos el 0,95, y el SRMR no alcanza el 0,03 , por lo que existe evidencia para el no rechazo de dichos tres modelos (Hair et al. 2006; Hu y Bentler 1999). 
Tabla 4.

Resultados del AFC: Modelo JDC(-S).

\begin{tabular}{ll|ccccc}
\hline \multicolumn{1}{c|}{ INDICADORES } & DC & DCL & CT & AP & $\mathrm{R}^{2}$ \\
\hline X1 & Trabajar muy rápido & $0,86^{* *}$ & & & & 0,74 \\
X2 & Plazos estrictos-cortos & $0,72^{* *}$ & & & & 0,52 \\
X3 & Trabajo intelectualmente exigente & & $0,86^{* *}$ & & & 0,75 \\
X4 & Trabajo emocionalmente exigente & & $0,78^{* *}$ & & & 0,61 \\
X5 & Puede elegir o modificar el orden de las tareas & & & $0,89^{* *}$ & & 0,79 \\
X6 & Puede elegir o modificar el método de trabajo & & & $0,87^{* *}$ & & 0,76 \\
X7 & Puede elegir o modificar el ritmo de trabajo & & & $0,90^{* *}$ & & 0,82 \\
X8 & Puede elegir o modificar la distribución/duración pausas & & & $0,81^{* *}$ & & 0,65 \\
X9 & Recibe ayuda compañeros & & & & $0,96^{* *}$ & 0,92 \\
X10 & Recibe ayuda superiores & & & & $0,68^{* *}$ & 0,46 \\
\hline & DIMENSIONES & DC & DCL & CT & AP & \\
\hline DC & DEMANDA CUANTITATIVA & 1,00 & & & & \\
DCL & DEMANDA CUALITATIVA & $0,17^{* *}$ & 1,00 & & & \\
CT & CONTROL & $-0,03$ & $0,37^{* *}$ & 1,00 & & \\
AP & APOYO & $0,21^{* *}$ & $0,14^{* *}$ & 0,02 & 1,00 & \\
\hline & Coeficiente de Fornell y Larcker (CVE) & 0,63 & 0,67 & 0,77 & 0,69 & \\
& Omega de McDonald (CFC) & 0,77 & 0,80 & 0,81 & 0,81 & \\
\hline
\end{tabular}

* Significativa al nivel 0,05 .

** Significativa al nivel 0,01 .

En cuanto a la Hipótesis 2, los resultados muestran que al introducir los componentes del Modelo JDC(-S) en la ecuación de regresión, el incremento de la varianza explicada es significativo en la variable criterio "riesgo percibido" $\left(\Delta R^{2}=0,053\right)$. La percepción de Demandas cuantitativas ( $B=0,331, p<0,01)$, el Control $(B=-0,044, p<0,01)$ y el Apoyo $(B=-0,091, p<0,01)$ explican el riesgo percibido. A mayor percepción de demanda cuantitativa mayor es el riesgo percibido por el trabajador de sufrir un accidente-enfermedad, mientras que cuanto mayor es el Control y el Apoyo, menor es dicha percepción de riesgo Por lo tanto, los resultados apoyan parcialmente dicha hipótesis, ya que las Demandas cualitativas no presentan un efecto significativo $(B=0,012, p>0,10)$. Complementariamente, el análisis de los coeficientes de regresión estandarizados nos proporciona información a ser destacada, mostrando que las Demandas cuantitativas percibidas juegan un papel más importante que el Control y el Apoyo a la hora de explicar la variable riesgo percibido, además que las percepciones de Apoyo son más relevantes, dos veces, que el Control percibido a efectos de explicar el riesgo. Paralelamente, destacar que mediante este modelo cabe valorar positivamente la validez nomológica de tres 
Tabla 5.

Resultados de los MEE-VL.

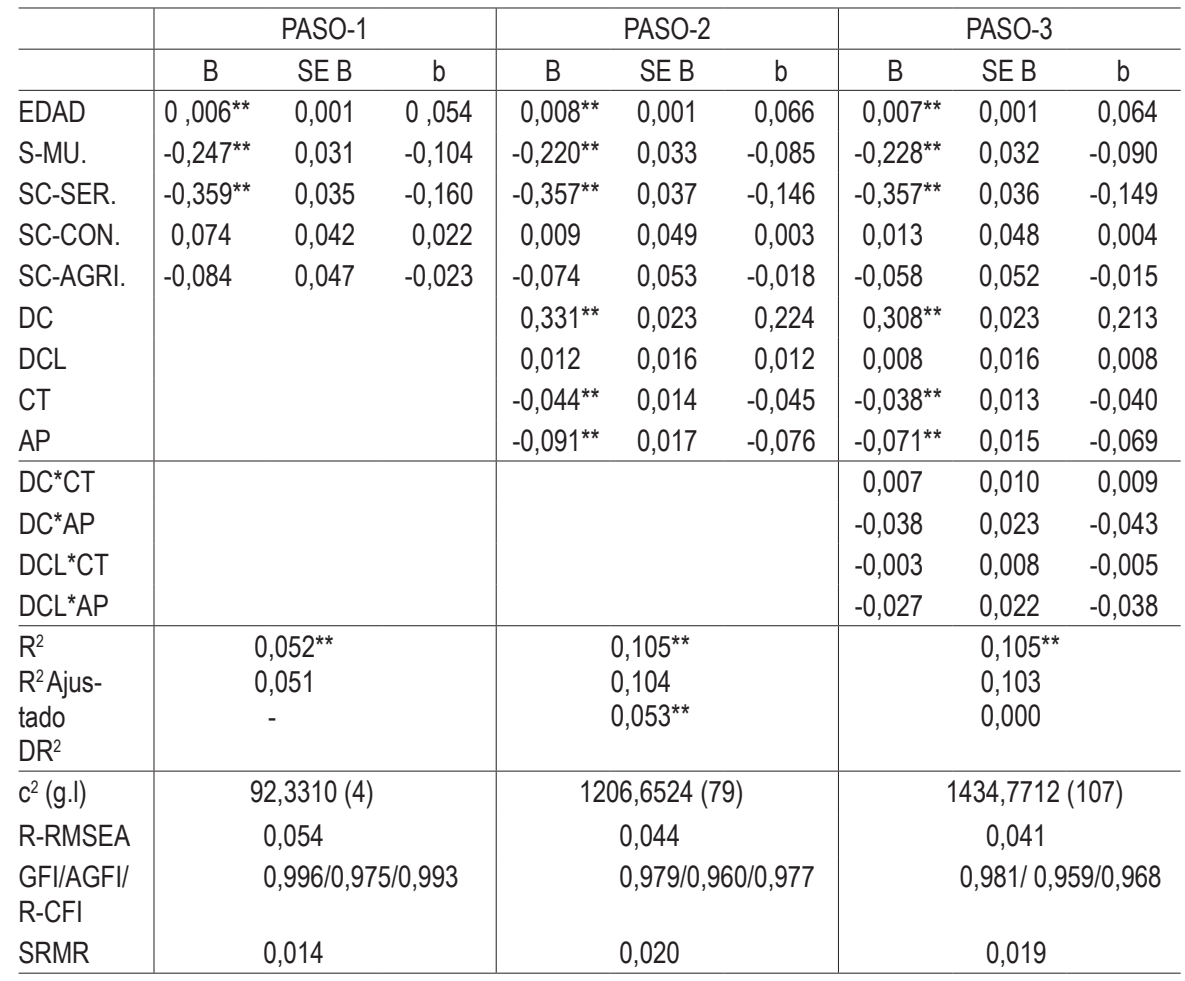

** Significativa al nivel 0,01 .

de los constructos representativos de los componentes del Modelo JDC(-S); Demandas Cuantitativas, Control y Apoyo a efectos de explicar el Riesgo percibido de un trabajador de sufrir un accidente-enfermedad.

Respecto a la Hipótesis 3, los resultados muestran que al introducir en la ecuación de regresión los potenciales efectos moduladores, tanto del Control como del Apoyo sobre ambos tipos de Demandas (Tabla 5), el incremento de la varianza explicada no es significativo en la variable criterio "riesgo percibido" $\left(\Delta R^{2}=0,000\right)$, por lo que se concluye que no existen efectos moduladores en la muestra analizada, e implica el rechazo de la Hipótesis 3 en sus dos variantes. En este punto, cabe reseñar que, si se comparan los efectos estimados por las variables explicativas a lo largo de los tres pasos del análisis de la regresión jerárquica (tres modelos), se aprecia una gran estabilidad en dichas estimaciones, únicamente se aprecian algunas diferencias en las centésimas, además, 
se mantiene la jerarquía de efectos observada desde el Paso 1 al Paso 3. Paralelamente, destacar, que los resultados obtenidos muestran evidencia de la importancia de las tres variables de control: sexo, edad y sector. Así, conforme aumenta la edad del individuo aumenta la percepción de riesgo, y que las mujeres y los trabajadores del sector servicios lo perciben en menor medida, aunque no ocurre lo mismo respecto a construcción-agricultura-industria. Por lo que los primeros resultados encontrados en el análisis bivariante entre los indicadores y las variables de control también se observan en términos del análisis multivariante realizado.

\section{Discusión}

El presente trabajo perseguía como objetivo determinar en qué medida el Modelo JDC(S) es de utilidad para explicar la percepción del Riesgo de enfermedad o accidente en el lugar de trabajo. Más específicamente, se trataba de analizar de qué modo los componentes de dicho modelo influyen en el Riesgo percibido de enfermedad o accidente, así como, analizar el papel del Control y del Apoyo como variables moduladoras entre las Demandas y el Riesgo percibido. Para ello, hubo que valorar previamente la estructura dimensional del modelo. Los resultados obtenidos proporcionan un apoyo parcial a las hipótesis planteadas.

En línea con la primera hipótesis, se ha encontrado que la componente de Demandas o estresores en el trabajo consta de dos dimensiones relacionadas pero independientes. Este resultado apoya los trabajos de autores como Martín et al. (2007); Mikkelsen, Ǿgaard, Landsbergis (2005); Kristensen (2002); Wall, Jackson y Mullarkey (1995), que defendían la diferenciación entre tipos de Demandas para mejorar la capacidad explicativa del modelo.

Sin embargo, la estructura encontrada de cuatro dimensiones no se sostiene cuando se analizan sus efectos sobre el Riesgo percibido, con la consideración de las variables de control, tal y como se había postulado en la hipótesis dos. Más específicamente, encontramos que las Demandas cualitativas no influyen en el riesgo percibido de sufrir un accidente-enfermedad. Sí se ha obtenido evidencia, en cambio, de la influencia positiva de las Demandas cuantitativas y negativa del Control y del Apoyo. Consecuentemente, la hipótesis dos tiene una aceptación parcial. No obstante, este resultado apoya también, lo encontrado en otras investigaciones en las que, al diferenciar entre Demandas, no todas eran predictivas de la variable a explicar (Martín et al. 2007; Mikkelsen, Ǿgaard, Landsbergis 2005). Una posible explicación para este resultado es que, aun cuando las exigencias de carácter emocional o intelectual puedan objetivamente generar estrés en los individuos, estos no llegan a percibir sus efectos perjudiciales sobre su salud física o psíquica.

A partir de aquí, era necesario determinar si el Control y el Apoyo tenían un efecto modulador sobre las Demandas cuantitativas. Esto ha sido puesto a prueba en el contrate de las hipótesis $3 a$ y $3 b$ y los resultados nos conducen a su rechazo. A diferencia 
de lo que se esperaba, el efecto de las Demandas sobre el Riesgo percibido no se ve reducido conforme aumenta el grado de Control o el de Apoyo, como en el caso anterior bajo la consideración de las variables de control. Por tanto, el estudio apoya la tendencia habitual, encontrada en los trabajos que emplean el Modelo JDC(-S), donde se confirma sistemáticamente la existencia de efectos aditivos-directos (ver estudios compilatorios de Häusser 2010; Van der Doeff y Maes 1999). El propio Häusser (2010) señala que sin embargo los efectos moduladores solo obtienen evidencia empírica, parcial o total, en el $30 \%$ de los estudios revisados en el período 1998-2009. Los resultados nos permiten concluir que los individuos perciben los efectos beneficiosos del Control y del Apoyo para su salud, pero no así el papel que estos recursos podrían tener para redefinir una situación estresante, debilitando así su efecto sobre la salud. En el caso del Control puede ocurrir que, aun cuando el individuo pueda tener mayor o menor discrecionalidad para organizar su trabajo, esto no evite la tensión derivada de exigencias del trabajo que vienen generalmente desde ámbitos que escapan de su control. Del mismo modo, la ausencia del efecto amortiguador del Apoyo podría deberse, bien a que el efecto beneficioso del apoyo solo funciona ante niveles elevados de estrés, bien a la discontinuidad con que el individuo puede recurrir a este recurso para enfrentar una situación estresante, bien al tipo de apoyo que el individuo puede encontrar, que no siempre se ajusta a la naturaleza de la ayuda que se requiere.

Estos resultados tomados en su conjunto suponen un cierto respaldo al Modelo JDC(-S). Concretamente, ponen de manifiesto con claridad la pertinencia de considerar aspectos como las Demandas cuantitativas, el grado de Control y el de Apoyo, junto con los otros factores individuales, contextuales y propios de la tarea desarrollada (Zimolong y Trimpop 1998) si queremos llegar a comprender variaciones en la manera como los empleados perciben el riesgo de sufrir un accidente o enfermedad como consecuencia de su actividad laboral.

La aplicación del Modelo JDC(-S) a una variable de percepción, de naturaleza contingente, nos permite ir más allá de las aplicaciones tradicionales de este Modelo basadas en la presencia real de problemas de salud, sea con base en diagnósticos técnicos (punto de vista objetivo), sea con base en autodiagnósticos de los trabajadores.

Futuras investigaciones deberán por un lado, prestar mayor atención a factores como la edad, el sexo y el sector de actividad, explorando la dimensionalidad del modelo y su utilidad para explicar percepciones de riesgo en el trabajo. Por otro lado, deberán considerar, junto con otro tipo de factores (Zimolong y Trimpop 1998), las influencias postuladas en el Modelo JDC(-S) con vistas al diseño de modelos más comprehensivos y de mayor validez predictiva.

Tal y como se ha puesto de manifiesto en este artículo, dada la importancia de que los trabajadores no tengan una percepción distorsionada del riesgo de salud para que se impliquen en una conducta más segura, de nuestro trabajo se desprenden varias implicaciones prácticas.

Primero, nuestros resultados nos han conducido a afirmar que las exigencias emocionales e intelectuales no influyen en la percepción del riesgo de salud del trabajador. 
Por otro lado, son numerosos los estudios sobre salud laboral donde se han obtenido evidencias objetivas del efecto estresante de dichas demandas. Estos dos hechos pueden generar un efecto distorsionador del riesgo de salud, percibiéndose un menor riesgo del que objetivamente cabría atribuir y, por ende, promover un comportamiento más imprudente. Sobre la base de estos resultados, cualquier empresa u organización -especialmente aquella en que este tipo de demandas son mayores (v. gr. profesión docente o sanitaria)-, debería preocuparse por conocer y valorar este tipo de demandas, así como establecer estrategias para gestionarlas adecuadamente, ya que, dado que el trabajador no percibe riesgo en ellas, es difícil que adopte activamente comportamientos que prevengan el efecto negativo sobre su salud.

Si bien, hay que añadir que, aun cuando esta dimensión cualitativa no ha resultado significativa en el modelo final, los análisis de las variables de control Sexo y Sector muestran el peso específico de este tipo de Demandas sobre el Riesgo percibido en el caso de las mujeres, así como en el sector servicios. Esto supondría una reorientación de las políticas de estrés según género y sector.

Una segunda implicación tiene que ver con la ausencia de efecto amortiguador del Control y del Apoyo sobre el riesgo de salud percibido. Una organización preocupada por mejorar la salud en el entorno de trabajo debería analizar las causas de esta percepción. Así, se podría trabajar en al menos dos frentes. En primer lugar, formando a los trabajadores en habilidades que les permitan un uso más eficaz de ambos recursos para afrontar la situación estresante (por ejemplo, enseñando cómo organizar mejor el trabajo o cómo buscar el apoyo de jefes y compañeros para desatascar cuellos de botella). En segundo lugar, también se podría disminuir el riesgo de salud si se promoviera una organización basada más en equipos de trabajo especializados donde se pueda contar con un tipo de apoyo centrado en la especificidad de la tarea y disponible de manera continua para el quehacer del trabajador.

No obstante, nuestro trabajo no está exento de limitaciones. En nuestra opinión, la principal tiene que ver con el instrumento de medida. El hecho de no haber empleado un cuestionario ad hoc ha limitado las posibilidades de utilización de un mayor número de ítem para medir las diferentes dimensiones del modelo. No obstante, esta limitación se ha intentado compensar mediante la aproximación metodológica empleada y con las evidencias bibliográficas sobre la validez de contenido de los ítem seleccionados. La metodología utilizada en el contraste de las hipótesis, tal y como se ha descrito, son los Modelos de Ecuaciones Estructurales con Variables Latentes, por lo que se ha considerado explícitamente el error de medición en los indicadores considerados mediante modelos de medida. Para finalizar, es necesario hacer notar que dicha limitación también es uno de sus puntos fuertes, el tamaño muestral resultante asciende a 7.512 registros completos, lo que permite reducir los errores de Tipo I y de Tipo II asociados a los contrastes de hipótesis. Por otro lado, se ha utilizado una encuesta que se realiza cada cuatro años, un período en el que las condiciones de trabajo pueden verse modificadas sustancialmente. Sin embargo, nuestro propósito no era tanto ofrecer una radiografía coyuntural del riesgo percibido en el lugar de trabajo, sino una medición más estructural 
de cómo se genera dicha percepción, lo cual no es probable que sufra modificaciones a corto o medio plazo.

A modo de conclusión, nos gustaría resaltar la aplicabilidad del Modelo JDC(-S) para estudiar aspectos de salud laboral no centrados exclusivamente en la presencia real del problema. En nuestro trabajo se ha mostrado la utilidad del modelo para explicar percepciones de riesgo subjetivo, lo que amplía las líneas de investigación hacia el análisis de la potencialidad percibida de cualquier problema de salud, aportando otros puntos de vista para el desarrollo de estrategias que promuevan un comportamiento más seguro de los trabajadores.

\section{Referencias Bibliográficas}

Aiken, L. S. y S. G. West. 1991. Multiple Regression: Testing and Interpreting Interactions. Newbury Park, CA: Sage.

Akerboom, S. y S. Maes. 2006. "Beyond demand and control: The contribution of organizational risk factors in assessing the psychological well-being of health care employees". Work \& Stress 20:21-36.

Alonso, E. y C. Pozo. 2002. "La percepción del riesgo en la prevención de accidentes laborales". Apuntes de Psicología 3:1-7.

Anderson, J. C., y D. W. Gerbing. 1988. "Structural equation modeling in practice: A review and recommended two-step approach". Psychological Bulletin 103:411-423.

Bagozzi, R. P. 1980. Causal Models in Marketing. North Carolina: John Wiley \& Sons, Inc.

Bagozzi, R. P. 2010. "Structural equation models are modelling tools with many ambiguities: Comments acknowledging the need for caution and humility in their use". Journal of Consumer Psychology 20:208-214.

Bakker A. B., E. Demerouti y W. Verbeke. 2004. "Using the job demands-resources model to predict burnout and performance". Human Resource Management 43:83-104.

Bentler, P. M. 1989-2006. EQS Structural Equations Program Manual. Encino, CA: Multivariate Software.

Bentler, P. M. y A. Satorra 2000. "Hierarchical Regression Without Phantom Factors". Structural Equation Modeling 7:287-291.

Blanch, J. M., M. Sahagún y G. Cervantes. 2010. "Estructura Factorial del Cuestionario de Condiciones de Trabajo". Revista de Psicología del Trabajo y de las Organizaciones 26:175-189.

Bollen, K. A. 1989. Structural Equations with Latent Variables. Nueva York: Wiley.

Browne M. W. y R. Cudeck. 1993. "Alternative Ways of Assessing Model Fit". Pp.136-162 in Testing Structural Equation Models. Newbury Park, CA: Sage.

Browne, M. W y R. Cudeck. 1989. "Single sample cross-validation indices for covariance structures". Multivariate Behavioral Research 24:445-455. 
Cabezas, C. 2000. La calidad de vida de los profesionales. Madrid: Materiales Formación Médica Continuada en Atención Primaria.

Castro, R., L. Campero y B. Hernández. 1997. "La investigación sobre apoyo social en salud: situación actual y nuevos desafíos". Revista de Saúde Pública 31:425-435.

Cohen, J., y P. Cohen. 1983. Applied Multiple Regression/Correlation Analysis for the Behavioral Sciences. $2^{\text {nd }}$ ed. Hillsdale, $\mathrm{NJ}$ : Lawrence Erlbaum Associates, Inc.

Cudeck, R. y M. W. Browne. 1983. "Cross-validation of covariance structures". Multivariate Behavioral Research 18:147-167.

Ministerio de la Presidencia de España. 1995. Agencia Estatal Boletín Oficial del Estado. Ley 31/1995, de 8 de noviembre, sobre Prevención de Riesgos Laborales. 269:32590-32611; 10 de noviembre de 1995. Madrid: Boletín Oficial del Estado.

Fillion, I., M. Tremblay, M. Truchon, D. Co^te, C. W. Struthers y R. Dupuis. 2007. "Job Satisfaction and Emotional Distress Among Nurses Providing Palliative Care: Empirical Evidence for an Integrative Occupational Stress-Model". International Journal of Stress Management 14:1-25.

Fornell, C. y D. F. Larcker. 1981. "Evaluating Structural Equation Models with Unobservable Variables and Measurement Error". Journal of Marketing Research 18:39-50.

García, A. 2006. "Percepción de riesgo, piedra angular psicosocial y formativa". Formación de Seguridad Laboral 85:27-33.

Gracia, F. J., I. Silla, J. M. Peiró y L. Fortes-Ferreira. 2006. "El estado del contrato psicológico y su relación con la salud psicológica de los empleados". Psicothema 18:256-262.

Griffin, J. M., B. A. Greiner, S. A. Stansfeld y M. Marmot. 2007. "The effect of self-reported and observed job conditions on depression and anxiety symptoms: A comparison of theoretical models". Journal of Occupational Health Psychology 12:334-349.

Grönlund, A. 2007. "More control less conflict? Job demand-control, gender and work-family conflict". Gender, Work and Organization 14:476-497.

Hair, J. F., W. C. Black, B. J. Babin, R. E. Anderson y R. L. Tatham. 2006. Multivariate Data Analysis. $6^{\text {th }}$ ed. Upper Saddle River, New Jersey: Prentice Hall.

Häusser, J. A., A. Mojzisch, M. Niesel y S. Schulz-Hardt. 2010. "Ten years on: A review of recent research on the Job Demand-Control (-Support) model and psychological well-being". Work \& Stress 24:1-35.

Hu, L. y P. M. Bentler. 1999. "Cutoff criteria for fit indexes in covariance structure analysis: Conventional criteria versus new alternatives". Structural Equation Modeling 6:1-55.

Instituto Andaluz de Prevención de Riesgos Laborales. 2008. 1. ${ }^{a}$ Encuesta Andaluza de Condiciones de trabajo. Sevilla: Ed. Junta de Andalucía, Consejería de Empleo.

ISTAS. 2004. "Identificación y evaluación de riesgos psicosociales". Madrid: Instituto Sindical de Trabajo, Ambiente y Salud. Consulta 21 de marzo de 2010 (http://www.istas.net/web/index. asp?idpagina=3185).

Janssen, O. 2000. "Job demands, perceptions of effort-rewards fairness and innovative work behavior". Journal of Occupational and Organizational Psychology 73:287-302. 
Johnson, J. V. y E. M. Hall. 1988. "Job Strain, Work place social support and cardiovascular disease: a cross-sectional study of a random sample of the Swedish working population". American Journal of Public Health 78:1336-1342.

Jöreskog, K. G. 1993. "Testing structural equation models", Pp. 294-316 en Testing Structural Equation Models. Newbury Park, CA.: Sage Publications.

Jöreskog, K. G., D. Sörbom. 1996. LISREL 8: User's Reference Guide. USA: SSI Scientific Software International.

Karasek, R. 1979. "Job demands, Job decision latitude and mental strain: Implications for job redesign". Administrative Science Quarterly 24:285-308.

Karasek, R. y T. Theorell. 1990. Healthy work: stress, productivity and the reconstruction of the working life. Nueva York: Ed. Basic Books.

Karasek. R. 1990. "Lower health risk with increased job control among white collar workers". Journal of Organizational Behaviour 11:171-185.

Kleinman, A., L. Eisenberg y B. Good. 1978. "Culture, illness and care: clinical lessons from anthropologic and cross-cultural research". Annals of internal Medicine 88:251-8.

Kristensen, T. y V. Borg. 2001. A new tool for assessing psychosocial factors at work: the Copenhagen psychosocial questionnaire. Proceedings from the Third European Academy of Occupational Health Psychology, 24-27 octubre, Barcelona.

Landsbergis, P. A. 1988. "Occupational Stress among health care workers: a test of a job demandscontrol model". Journal of Organizational Behavior 9:217-240.

(De) Lange A. H., T. W. Taris, M. A. J. Kompier, I. L. D. Houtman y P. M. Bongers. 2002. "Effects of stable and changing demand-control histories on worker health". Scandinavian Journal of Work, Environment \& Health 28:94-108.

López Araujo, B., A. Osca Segovia y J. M. Peiró. 2007. "El papel modulador de la implicación con el trabajo en la relación entre el estrés y la satisfacción laboral". Psicothema 19:81-87.

MacDonald, L. A., R. Karasek, L. Punnett, y T. Scharf. 2001. "Covariation between workplace physical and psychosocial stressors: evidence and implications for occupational health research and prevention". Ergonomics 44:696-718.

Martín, J. M., L. Luceño, M. Jaén y S. Rubio. 2007. "Relación entre factores psicosociales adversos, evaluados a través del cuestionario multidimensional Decore, y salud laboral deficiente". Psicothema 19:95-101.

Martín, P., M. Salanova y J. M. Peiró. 2007. "Job demands, job resources and individual innovation at work: Going beyond Karasek's model”. Psicothema 19:621-626.

McDonald, R. P. 1985. Factor analysis and related methods. Hillsdale. New Jersey: Lawrence Erlbaum Associates, Publishers.

Meliá, J. L. y M. Becerril. 2007. "Psychosocial sources of stress and burnout in the construction sector: A structural equation model". Psicothema 19:679-686.

Mikkelsen, A., T. Øgard y P. Landsbergis. 2005. "The effects of new dimensions of psychological job demands and job control on active learning and occupational health". Work \& Stress 19:153-175. 
Muthén, L. K. y B. O. Muthén. 1998-2007. Mplus User's Guide. Fifth Edition. Los Angeles, CA: Muthén and Muthén.

Nunnally, J. C. y I. H. Bernstein. 1994. Psychometric Theory. New York: McGraw-Hill.

Pelfrene, E., P. Vlerick, R. P. Mak, P. Smet, M. Kornitzer y G. Backer. 2001. "Scale reliability and validity of the Karasek' job demand-control-support' model in the Belstress study". Work \& Stress 15:297-313.

Pérez, J. y F. Martín. 1997. "El apoyo social". Nota técnica del Instituto Nacional de Seguridad e Higiene en el trabajo NTP 439.

Portell, M., M. D. Riba y R. Payés. 1997. "La definición de riesgo: implicaciones para su reducción". Revista de Psicología de la Salud 9:3-27.

Quick, J. C., J. D. Quick, D. L. Nelson y J. J. Hurrell. 1997. Preventive Stress Management in Organizations. Washington, DC: American Psychological Association.

Rafferty, Y., R. Friend y P. Landsbergis. 2001. "The association between job skill discretion, decision authority and burnout". Work \& Stress 15:73-85.

Sanne, B., A. Mykletun, A. A. Dahl, B. E. Moen y G. S. Tell. 2005. "Testing the Job Demand-ControlSupport model with anxiety and depression as outcomes: the Hordaland Health Study". Occupational Medicine 55:463-473.

Sargent, L y D. Terry. 2000. "The moderating role of social support in Karasek's job strain model". Work \& Stress 14:245-261.

Satorra, A. y P. M. Bentler. 1994. "Corrections to test statistics and standard errors in covariance structure analysis". Pp. 349-419 en Latent Variables Analysis: Applications for Developmental Research. Thousand Oaks, CA: Sage.

Satorra, A. 2003. "Power of chi-square Goodness-of-fit test in structural equation models: the case of non-normal data". Pp. 57-68 en New Developments of Psychometrics. Tokyo: Springer Verlag.

Schaufeli, W. y A. Bakker. 2004. "Job demands, job resources, and their relationship with burnout and engagement: a multi-sample study". Journal of Organizational Behavior 25:293-315.

Sjöberg, L. 2000. "Factors in risk perception". Risk Analysis 20:1-11.

Sjöberg, L. 2002. "Are received risk perception models alive and well?" Risk Analysis 22:665-669.

Tucker, J. S., R. R. Sinclair, C. D. Mohr, A. B. Adler, J. L. Thomas y A. D. Salvi. 2008. "A temporal investigation of the direct, interactive, and reverse relations between demand and control and affective strain". Work \& Stress 22:81-95.

Van der Doef, M. y S. Maes. 1999. "The Job Demand-Control (-Support) model and psychological wellbeing: a review of 20 years of empirical research". Work \& Stress 13:87-114.

Van Vegchel, N., J. Jonge y P. Landsbergis. 2005. "Occupational stress in (inter)action: the interplay between job demands and job resources". Journal of Organizational Behavior 26:535-560.

Vermeulen, M. y C. Mustard. 2000. "Gender differences in job strain, social support at work, and psychological distress". Journal of Occupational Health Psychology 5:428-440.

Wall, T. D., P. R. Jackson y S. Mullarkey. 1995. "Further evidence on some new measures of job control, cognitive demands and production responsibility". Journal of Organizational Behavior 16:431-455. 
Wall, T. D., P. R. Jackson, S. Mullarkey y S. K. Parker. 1996. "The demands-control model of job strain: a more specific test". Journal of Occupational and Organizational Psychology 69:153-166.

Wong, S., G. de Santics y N. Staudemayer. 2007. "The relationship between task interdependency and role stress: a revisit of the job demands-control model". Journal of Management Studies 44:284303.

Zimolong, B. y R. Trimpop. 1998. "Risk perception". Encyclopaedia of Occupational Health and Safety, vol. 2, 25-32. Geneva: ILO.

María José Montero Simó es Profesora titular del área de Comercialización e Investigación de Mercados en la Universidad Loyola Andalucía. Directora del Departamento de Gestión Empresarial y Métodos Cuantitativos. Doctora en Investigación y Técnicas de Mercado por la Universidad de Córdoba. Licenciada en Ciencias Económicas y Empresariales (Sección Empresariales) por la Universidad de Córdoba. Master en Dirección de Marketing y Gestión Comercial, por la Escuela Superior de Gestión Comercial y Marketing (ESIC). Sus áreas de especialización son: el Marketing Social, la comercialización para el desarrollo y la Responsabilidad Social de las empresas (RSE). Coordinadora del Grupo de Investigación en Ética y Responsabilidad Social de ETEA (Grupo ERES).

Pilar Rivera Torres es Profesora Titular en el Área de Comercialización e Investigación de Mercados en la Facultad de Economía y Empresa de la Universidad de Zaragoza. Pertenece al Grupo de Investigación CREVALOR reconocido por la Dirección General de Aragón y ha publicado sus trabajos en diferentes revistas, tanto nacionales como internacionales. Entre sus logros cabe destacar las siguientes revistas en las que ha publicado sus trabajos: Environmental Management, Environment And Planning A, Strategic Management Journal, Total Quality Management \& Business Excellence, Journal of Cleaner Production, Annals of Forest Science, Management Decisión, Supply Chain Management-An International Journal y International Journal of Environmental Research and Public Health.

Rafael Ángel Araque Padilla es Profesor titular del área de Comercialización e Investigación de Mercados en la Universidad Loyola Andalucía. Doctor en Investigación y Técnicas de Mercado por la Universidad de Córdoba. Licenciado en Ciencias Económicas y Empresariales (Sección Empresariales) por dicha universidad. Master en Dirección de Marketing y Gestión Comercial por ESIC. Sus áreas de especialización son el Marketing Social, la comercialización para el desarrollo y la Responsabilidad Social de la Empresa (tesis doctoral), Coordinador del Grupo de Investigación en Ética y Responsabilidad Social de ETEA (Grupo ERES), y miembro del Consejo de Redacción de la Revista de Fomento Social.

RECIBIDO: $26 / 07 / 12$

ACEPTADO: 26/07/12 